This item was submitted to Loughborough's Research Repository by the author.

Items in Figshare are protected by copyright, with all rights reserved, unless otherwise indicated.

Scaling relations for implantation of size-selected $\mathrm{Au}, \mathrm{Ag}$, and Si clusters into graphite

PLEASE CITE THE PUBLISHED VERSION

PUBLISHER

(C) American Physical Society

LICENCE

CC BY-NC-ND 4.0

REPOSITORY RECORD

Pratontep, S., P. Preece, C. Xirouchaki, R.E. Palmer, Carlos F. Sanz-Navarro, Steven D. Kenny, and Roger Smith. 2019. "Scaling Relations for Implantation of Size-selected Au, Ag, and Si Clusters into Graphite". figshare. https://hdl.handle.net/2134/1744. 


\title{
Scaling Relations for Implantation of Size-Selected Au, Ag, and Si Clusters into Graphite
}

\author{
S. Pratontep, P. Preece, C. Xirouchaki, and R. E. Palmer \\ Nanoscale Physics Research Laboratory, School of Physics and Astronomy, The University of Birmingham, \\ Birmingham B15 2TT, United Kingdom \\ C. F. Sanz-Navarro, S. D. Kenny, and R. Smith \\ School of Mathematics and Physics, Loughborough University, Loughborough LE11 3TU, United Kingdom
}

(Received 29 July 2002; published 5 February 2003)

\begin{abstract}
The deposition of size-selected clusters represents a new route to the fabrication of truly nanometerscale surface architectures, e.g., nanopores. We report a systematic experimental study, coupled with molecular dynamics simulations, of the implantation depths of size-selected $\mathrm{Au}_{7}, \mathrm{Ag}_{7}$, and $\mathrm{Si}_{7}$ clusters in the model graphite substrate. For impact energies between 1.0 and $5.5 \mathrm{keV}$, we find that the implantation depth scales linearly with the momentum of the clusters for all three types of cluster. This "universal" behavior is consistent with a (viscous) retarding force proportional to the velocity of the cluster, akin to Stokes's law.
\end{abstract}

DOI: 10.1103/PhysRevLett.90.055503

Ion-surface interactions play a crucial role in many surface processes and their applications [1-3]. When an atomic ion is incident upon a solid surface, the implantation depth depends upon the detailed impact parameters, e.g., the impact site within the surface unit cell, so that a distribution of depths results [4]. If the projectile ion is an atomic cluster larger than the surface unit cell, narrower depth distributions can be anticipated. For $\mathrm{Ag}_{3}{ }^{-}$ions incident upon the model graphite surface, experiments and molecular dynamics (MD) simulations show that the implantation depth depends on the impact site and the orientation of the cluster as well as the kinetic energy [4]. However, when the cluster size is increased to $n=7$ the implantation depth can be defined to within only one atomic layer of the substrate; the mean diameter of $\mathrm{Ag}_{7}$ clusters, $\sim 5.4 \AA$ [5], already exceeds the unit cell size in graphite, $2.42 \AA$. Such a precise definition of the implantation depth for size-selected atomic clusters suggests the creation of novel nanoscale architectures, which may serve as model catalysts [6], prototypical binding sites to immobilize [7] biological molecules or nanoscale pores for DNA sequencing [8], or to create "molecular corrals" [9] by lateral etching.

The creation of such well-defined model nanostructures by the deposition of size-selected clusters requires us to develop a detailed understanding of the clustersurface interaction [10], especially the scaling relations which govern cluster impact and implantation. In the case of the $\mathrm{Ag}_{\mathrm{N}} /$ graphite system, significant progress has recently been reported [11-14]. For cluster sizes in the range $n=50-200$, the threshold energy for "pinning" of the incident cluster to the point of impact scales linearly with the cluster size. This process enables nanostructured surfaces to be fabricated which are stable against cluster diffusion even at room temperature and in the ambient [11]. Further, MD simulations for the size range $n=$
PACS numbers: 61.46.+w, 68.55.Ln, 79.20.Rf, 81.05.Uw

20-200 indicate an implantation depth, $D$, which scales linearly with the kinetic energy, $E$, and inversely with the cross-sectional area of the cluster; $D \propto E / N^{2 / 3}$ [12]. However, first experimental measurements for (smaller) $\mathrm{Ag}_{7}{ }^{-}$clusters [13] indicate that the implantation depth scales with velocity, not energy, a difference which is also reflected in MD results [14]. In order to shed further light on the intriguing dynamics of the cluster-surface impact, we report in this Letter a systematic experimental study of the implantation depth of three different kinds of clusters, including both metal clusters, $\mathrm{Au}_{7}$ and $\mathrm{Ag}_{7}$, and semiconductor clusters, $\mathrm{Si}_{7}$, into the graphite surface. The results show that, for elevated kinetic energies, the implantation depth scales with the momentum of the clusters in all cases. This universal behavior identified will enable the fabrication of precisely defined metallic and semiconductor nanostructures.

The size-selected clusters in this study were produced by two methods: with an rf magnetron sputtering, gas aggregation cluster source $[11,15]$ in the case of $\mathrm{Ag}_{7}{ }^{+}$, $\mathrm{Au}_{7}{ }^{+}$, and $\mathrm{Si}_{7}{ }^{+}$clusters; and with a cesium sputtering source [16] for $\mathrm{Ag}_{7}{ }^{-}$and $\mathrm{Au}_{7}{ }^{-}$. The clusters were deposited in high vacuum $\left(\sim 10^{-7}\right.$ mbar) onto highly oriented pyrolytic graphite (HOPG) samples, cleaved shortly before introduction to the vacuum. The impact energy of the clusters (in the range 1.0-5.5 keV) was determined by a bias voltage applied to the sample during deposition. The cluster implantation depths (more specifically, the damage track depths) were obtained by the etching method [17-23]. Thus the samples were etched in air at $650{ }^{\circ} \mathrm{C}$ for a duration of 3-5 min immediately after removal from the vacuum. The depth of the etch pits was measured using a bench-top scanning tunneling microscope (STM). The STM images were obtained with mechanically cut $\mathrm{Pt} / \mathrm{Ir}$ tips; typical imaging parameters were a tip bias of $0.3-0.5 \mathrm{~V}$ and a tunneling current of $0.3-0.5 \mathrm{nA}$. 
Figure 1(a) is a representative STM image showing the hexagonal etch pits formed by anisotropic lateral etching of a graphite sample implanted with $\mathrm{Si}_{7}{ }^{+}$ions at $4.5 \mathrm{keV}$. Each cluster track generates an etch pit. Figure 1(b) shows a height profile along the white line marked in Fig. 1(a). From such data a distribution of etch pit depths for each cluster $\left(\mathrm{Si}_{7}, \mathrm{Ag}_{7}\right.$, and $\left.\mathrm{Au}_{7}\right)$ and each kinetic energy was obtained. Figure 2 shows graphs of the depth distributions for $\mathrm{Si}_{7}$ implantation at 1.0, 2.5, and $4.5 \mathrm{keV}$ (i.e., Fig. 1). The width of these distributions arises from a competition between lateral etching and defect annealing during the etching procedure; the cluster implantation depth corresponds to the deepest pits obtained for each combination of cluster composition and kinetic energy, extracted according to the recipe of Ref. [13], which gives excellent agreement with the results of MD simulations.

Figure 3 presents a compilation of the extensive data sets obtained for the implantation depths of $\mathrm{Si}_{7}, \mathrm{Ag}_{7}$, and $\mathrm{Au}_{7}$ clusters over the kinetic energy range $0.5-5.5 \mathrm{keV}$. To within experimental error, the charge of the $\mathrm{Au}$ and $\mathrm{Ag}$ clusters (positive or negative) was found to have no effect upon the measured depth. Thus electronic braking [1] of the clusters plays a negligible role in these circumstances. To explore fully the appropriate scaling relations connecting the implantation depth to the dynamical parameters characterizing the incident clusters, Fig. 3 plots the experimental implantation depths as a function of three different parameters, the kinetic energy, Fig. 3(a), the velocity, Fig. 3(b), and the momentum, Fig. 3(c), of the clusters. Inspection of the graphs reveals immediately
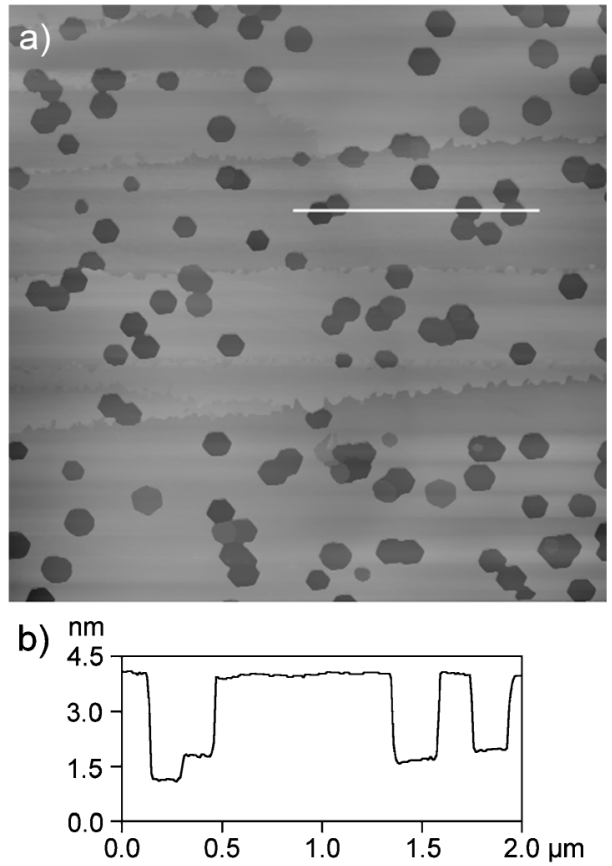

FIG. 1. (a) STM image $\left(5 \times 5 \mu \mathrm{m}^{2}\right)$ of the graphite surface showing etch pits created by oxidative etching after $\mathrm{Si}_{7}{ }^{+}$ cluster implantation at $4.5 \mathrm{keV}$. (b) Height profile corresponding to the white line in (a). that, in the case of the Si clusters, a linear scaling is not obtained in any case. We return to this case below. For the $\mathrm{Au}$ and $\mathrm{Ag}$ clusters, comparison between Figs. 3(a) and 3 (b) indicates that, in both cases, there is a linear relationship between the implantation depth and the velocity, not the kinetic energy, of the clusters (though the slopes are different). These results confirm the velocity scaling reported in the preliminary measurements of $\mathrm{Ag}_{7}{ }^{-} \mathrm{im}-$ plantation $[13,14]$ and show that the same type of velocity scaling also applies to the $\mathrm{Au}_{7}$ clusters (but not, at first sight, $\mathrm{Si}_{7}$ ).

While Fig. 3(b) demonstrates that, for both $\mathrm{Ag}_{7}$ and $\mathrm{Au}_{7}$ clusters, the measured implantation depth $(D)$ is linearly related to the cluster velocity, the different slopes indicate that at least one other factor must also play a role in the dynamics. Inspection of Fig. 3(c), in which the same implantation depth data are plotted against momentum $(p)$, shows that in this case the slopes of the $\mathrm{Au}$ and $\mathrm{Ag}$ curves [24] are practically identical $(0.147 \pm$ $0.015 \mathrm{fsec} \mathrm{u}^{-1}$ and $0.139 \pm 0.009 \mathrm{fsec} \mathrm{u}^{-1}$, respectively). Thus the fundamental scaling relation, which unites the data for the Au and $\mathrm{Ag}$ clusters, is $D \propto p$. The nature of this scaling relationship also sheds light upon the nature of the force $(F)$ experienced by the cluster in the graphite substrate; specifically, it is consistent with a braking force $F$ which is proportional to the normal velocity $(v)$ of the cluster, akin to Stokes's law. To prove this, suppose that $F \approx-b v$, where $b$ is a constant. Then, integrating Newton's second law, from initial velocity $v_{0}$ at the point (above the surface) where the interaction starts $(x=$ const) to $v=0$ at depth $x=D$, yields simply

$$
D=\frac{m v_{0}}{b}+\text { const. }
$$

The linear relationship which results is consistent with the data for $\mathrm{Au}_{7}$ and $\mathrm{Ag}_{7}$ clusters, Fig. 3(c). The value of the constant will depend on the diameter of the clusters, since the interaction begins a distance above the surface

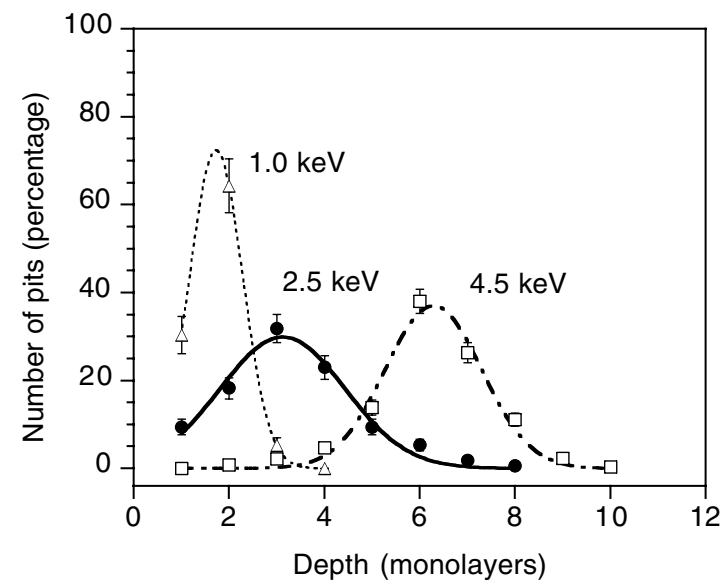

FIG. 2. The distribution of the etch pit depths obtained after implantation of $\mathrm{Si}_{7}^{+}$clusters into graphite at 1.0, 2.5, and $4.5 \mathrm{keV}$. The data are fitted to Gaussian distributions. 

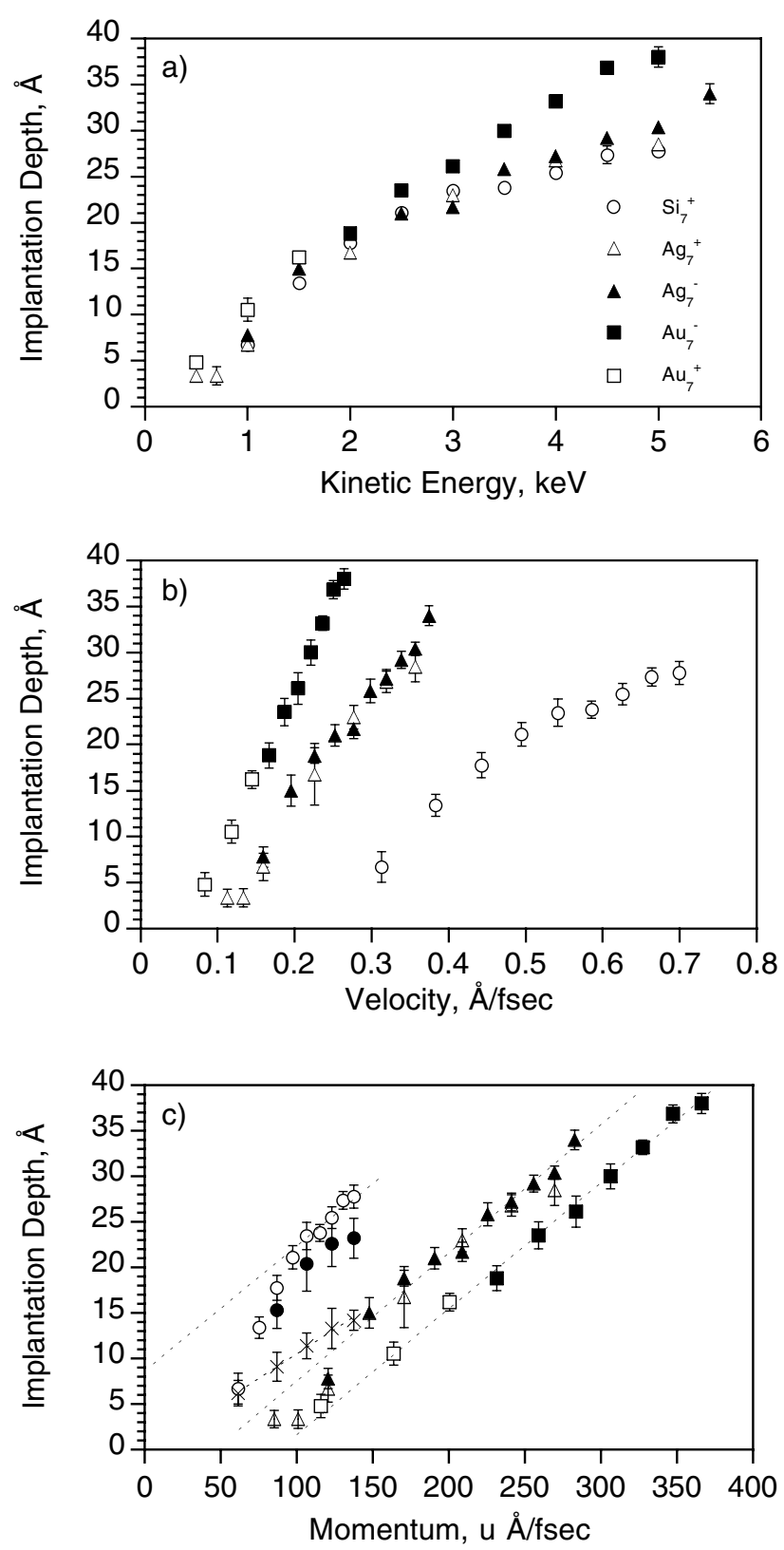

FIG. 3. The implantation depths for $\mathrm{Au}_{7}, \mathrm{Ag}_{7}$, and $\mathrm{Si}_{7}$ clusters in graphite as a function of (a) kinetic energy, (b) velocity, and (c) momentum. The depths are given with respect to the topmost graphite layer. The data for $\mathrm{Ag}_{7}{ }^{-}$are obtained from Ref. [13]. In (c), the dashed lines are the best fit straight lines for $\mathrm{Au}_{7}$ and $\mathrm{Au}_{7}$ (see text). The filled circles are the depths of the damage tracks from MD simulations for $\mathrm{Si}_{7}$. The crosses are the calculated center-of-mass cluster implantation depths.

of the order of the cluster radius. However, $\mathrm{Au}_{7}$ and $\mathrm{Ag}_{7}$ clusters have similar size, and the different (extrapolated) intercepts most likely relate to the threshold energy required to create the first defect in the top graphite layer (equivalent to the pinning energy discussed in Ref. [11]).

We have noted already that the measured behavior of the $\mathrm{Si}_{7}$ clusters, Fig. 3(c), does not follow the same linear dependence on momentum which is true of $\mathrm{Au}_{7}$ and $\mathrm{Ag}_{7}$. Indeed, Fig. 3 shows that the $\mathrm{Si}_{7}$ implantation depths do not simply scale with any of the dynamical parameters plotted. However, closer inspection of Fig. 3(c) shows that, for higher energies (momenta), the Si data can be represented by a straight line with the same slope as the $\mathrm{Au}$ and Ag data. Thus, in the high energy regime, there is a universal behavior which unites the metal clusters, $\mathrm{Au}_{7}$ and $\mathrm{Ag}_{7}$, with the semiconductor cluster, $\mathrm{Si}_{7}$. It appears that the same (viscous) retarding force, Eq. (1), operates in all cases. Why then should silicon depart from this common behavior at lower energies?

To explore further the behavior of the $\mathrm{Si}_{7}$ clusters, we have conducted MD simulations [14] of their implantation into graphite. The Brenner potential [25] was employed for graphite, together with interlayer terms [26], while $\mathrm{Si}$ and $\mathrm{SiC}$ were treated by the Tersoff potential $[27,28]$. The Ziegler-Biersack-Littmark (ZBL) screened Coulomb potential [29] was employed to represent the short range repulsive potential. Figure 4 shows MD simulations for $\mathrm{Ag}_{7}$ and $\mathrm{Si}_{7}$ clusters deposited at $4 \mathrm{keV}$ after completion of the collisional phase of the cascade. One key point which emerges from the simulations is that, in the case of $\mathrm{Si}$ (but not $\mathrm{Ag}$ ), the damage track in the graphite substrate extends a few layers below the cluster itself, attributed to the creation of high energy carbon recoils. Since the etching method measures the depth of the damage track, and not the implantation depth of the cluster itself, the MD simulations imply that the data of Figs. 2 and 3 will overestimate the true implantation depths for silicon. Thus in Fig. 3(c) we plot the depth of the etch pits for $\mathrm{Si}_{7}$ predicted from the MD simulations for four different energies, 2, 3, 4, and $5 \mathrm{keV}$ (filled circles). The agreement with the experimental etch pit depths is very good. We also plot in Fig. 3(c) the (center of mass) implantation depths of the $\mathrm{Si}_{7}$ clusters extracted from the MD simulations. The slope of the line through these points is $0.108 \pm 0.024 \mathrm{fsec} \mathrm{u}^{-1}$. This is in rather good agreement with the experimental slopes for $\mathrm{Ag}_{7}$ and $\mathrm{Au}_{7}$. We conclude that the $\mathrm{Si}_{7}$ clusters follow the same
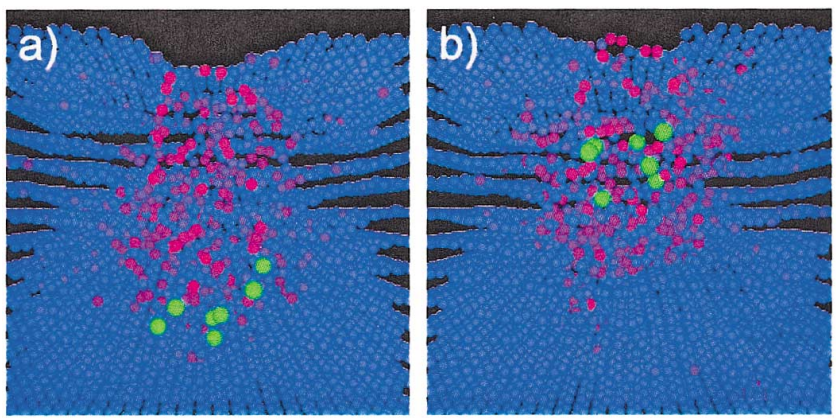

FIG. 4 (color). Snapshot from MD simulations of cluster implantation into graphite, after completion of the collisional phase, for normally incident, seven-atom clusters at $4 \mathrm{keV}$. (a) $\mathrm{Ag}_{7}$ cluster; (b) $\mathrm{Si}_{7}$ cluster. In (b) there is more damage underneath the implanted cluster, caused by recoil $\mathrm{C}$ atoms. Note also the sputtered $\mathrm{C}$ atom above the surface in this case. The cluster atoms are shown in green. 
universal scaling behavior as the metal clusters and that the "anomalous" behavior of the experimental etch pit depths arises from the creation of energetic carbon recoils which extend the depth of the damage track.

Why should the creation of the energetic carbon recoils be different for $\mathrm{Si}_{7}$ than $\mathrm{Au}_{7}$ and $\mathrm{Ag}_{7}$ ? The reason is a pure mass effect. Our MD simulations show that the covalent attraction between the $\mathrm{C}$ and $\mathrm{Si}$ atoms (the $\mathrm{Si}$ $\mathrm{C}$ bond energy is $4.6 \mathrm{eV}$ in $\mathrm{SiC}$ [30], while the $\mathrm{Ag}$ adsorption energy on graphite lies in the range 0.23 to $0.54 \mathrm{eV}$ [31]) has a negligible effect on the implantation depths. If the mass of the Si atom is artificially increased to that of $\mathrm{Ag}$, but with the covalent $\mathrm{Si}-\mathrm{C}$ interaction unchanged, almost exactly the same results are found as for Ag itself [14]. The mass effect is illustrated by the fact that the maximum energy transfer in a binary collision between a $\mathrm{Ag}$ atom of incident energy $E_{0}$ and a $\mathrm{C}$ atom at rest is $0.36 E_{0}$, whereas for an incident $\mathrm{Si}$ atom it is $0.84 E_{0}$. Thus higher energy carbon recoils can be created by the $\mathrm{Si}$ cluster, and some of these recoils cause damage deeper into the graphite substrate than the cluster itself. This effect saturates, Fig. 3(c), at high energies (momenta).

In summary, we have reported a systematic experimental study of the implantation depths of size-selected $\mathrm{Au}_{7}$, $\mathrm{Ag}_{7}$, and $\mathrm{Si}_{7}$ clusters in the model graphite substrate. A strikingly uniform behavior is observed, particularly at elevated impact energies, where the implantation depth is found to scale linearly with the momentum of the clusters in all three cases. We have also shown that this behavior is consistent with a retarding force proportional to the velocity of the cluster, akin to Stokes's law. In the case of $\mathrm{Si}_{7}$, the measured depth of the damage track exceeds the true cluster implantation depth, because of the creation of energetic recoil atoms. The data and model presented establish a "universality" in the implantation behavior of size-selected clusters which can be exploited to create well-defined, truly nanometer-scale architectures ("cluster down a well") for applications in single molecule physics, chemistry, and biology. In this case the lateral dimensions of the "pore" will be controlled by the cluster size and the depth determined by the kinetic energy, via the scaling relations which have been established. Future work needs to establish experimentally whether larger clusters (10-1000 atoms) follow the same scaling relations.

This work was supported by the EPSRC. S. P. acknowledges financial support from the DPST (Thailand), from the ORS Committee, and from the University of Birmingham. We are very grateful to Dr. D. J. Kenny for helpful discussions, to S. C. Weller and Dr. M. Couillard for their assistance in producing some of the $\mathrm{Au}_{7}{ }^{-}$cluster samples, and to Dr. F. Claeyssens for his contribution to the STM work.
[1] R. Smith, Atomic and Ion Collisions in Solids and at Surfaces (Cambridge University Press, Cambridge, U.K., 1997).

[2] T. S. Wand et al., Appl. Phys. Lett. 77, 3586 (2000); P. M. Voyles, D. A. Muller, J. L. Grazul, P. H. Citrin, and H. J. L. Gossmann, Nature (London) 416, 826 (2002)

[3] G. Costantini et al., Phys. Rev. Lett. 86, 838 (2001); S. Rusponi et al., Phys. Rev. Lett. 81, 4184 (1998).

[4] S. J. Carroll et al., Phys. Rev. Lett. 81, 3715 (1998).

[5] R. Fournier, J. Chem. Phys. 115, 2165 (2001).

[6] S. Abbet et al., Phys. Rev. Lett. 86, 5950 (2001); C. R. Henry, Surf. Sci. Rep. 31, 235 (1998); M. Valden, X. Lai, and D.W. Goodman, Science 281, 1647 (1998); U. Heiz et al., J. Am. Chem. Soc. 121, 3214 (1999).

[7] M. B. Viani et al., Nat. Struct. Biol. 7, 644 (2000).

[8] J. Li et al., Nature (London) 412, 166 (2001).

[9] D. L. Patrick, V. J. Cee, and T. P. Beebe, Science 265, 231 (1994).

[10] C. Brechignac et al., Phys. Rev. Lett. 88, 196103 (2002); B. Kaiser et al., Phys. Rev. Lett. 83, 2918 (1999); J. Schmelzer et al., Phys. Rev. Lett. 88, 226802 (2002).

[11] S. J. Carroll et al., J. Chem. Phys. 113, 7723 (2000); M. Helmer, Nature (London) 408, 531 (2000).

[12] S. J. Carroll et al., Phys. Rev. Lett. 84, 2654 (2000).

[13] D. J. Kenny et al., J. Phys. Condens. Matter 14, L185 (2002).

[14] C. F. Sanz-Navarro et al., Phys. Rev. B 65, 165420 (2002).

[15] S. J. Carroll, Ph.D. thesis, The University of Birmingham, 1999; B. von Issendorff and R. E. Palmer, Rev. Sci. Instrum. 70, 4497 (1999).

[16] S. G. Hall et al., Rev. Sci. Instrum. 68, 3335 (1997).

[17] H. Chang and A. J. Bard, J. Am. Chem. Soc. 113, 5588 (1991).

[18] J. R. Hahn and H. Kang, Surf. Sci. 446, L77 (2000).

[19] D. J. Kenny et al., Eur. Phys. J. D 16, 115 (2001).

[20] C. T. Reimann et al., Nucl. Instrum. Methods Phys. Res., Sect. B 140, 159 (1998).

[21] G. Bräuchle et al., Appl. Phys. Lett. 67, 52 (1995).

[22] R. P. Webb et al., Nucl. Instrum. Methods Phys. Res., Sect. B 122, 318 (1997).

[23] H. Hövel et al., Phys. Rev. Lett. 81, 4608 (1998).

[24] The slopes were obtained by a least squares fit to all the data points above a kinetic energy of $1 \mathrm{keV}$ for both the $\mathrm{Au}$ and Ag clusters.

[25] D.W. Brenner, Phys. Rev. B 42, 9458 (1990); 46, 1948 (1992).

[26] R. Smith and K. Beardmore, Thin Solid Films 272, 255 (1996).

[27] J. Tersoff, Phys. Rev. B 42, 9458 (1990).

[28] K. Beardmore and R. Smith, Philos. Mag. 74, 1439 (1996).

[29] J. F. Ziegler, J. P. Biersack, and U. Littmark, The Stopping and Range of Ions in Solids (Pergamon, New York, 1985).

[30] K. P. Huber and G. Herzberg, Molecular Spectra and Molecular Structure IV: Constants of Diatomic Molecules (Van Nostrand Reinhold, New York, 1979), p. 594.

[31] D. M. Duffy and J. A. Blackman, Surf. Sci. 415, L1016 (1998). 